\title{
The Structure and Methylation Level of the McMYB10 Promoter Determine the Leaf Color of Malus Crabapple
}

Ji Tian'

Department of Plant Science and Technology, Beijing University of Agriculture, Beijing 102206, China; and Beijing Collaborative Innovation Center for Eco-environmental Improvement with Forestry and Fruit Trees, Beijing 102206, China

Ke-ting Li ${ }^{1}$ and Shi-ya Zhang ${ }^{1}$

Department of Plant Science and Technology, Beijing University of Agriculture, Beijing 102206, China

Jie Zhang and Ting-ting Song

Department of Plant Science and Technology, Beijing University of Agriculture, Beijing 102206, China; and Beijing Collaborative Innovation Center for Eco-environmental Improvement with Forestry and Fruit Trees, Beijing 102206, China

\section{Yong-jun Zhu}

Department of Plant Science and Technology, Beijing University of Agriculture, Beijing 102206, China

Yun-cong $\mathbf{Y a o}^{2}$

Department of Plant Science and Technology, Beijing University of Agriculture, Beijing 102206, China; and Beijing Collaborative Innovation Center for Eco-environmental Improvement with Forestry and Fruit Trees, Beijing 102206, China

Additional index words. R6 and R1 minisatellites, anthocyanins, promoter structure, coloration

Abstract. Anthocyanins are protective pigments that accumulate in plant organs such as fruits and leaves, and are nutritionally valuable components of the human diet. The MYB10 transcription factor (TF) plays an important role in regulating anthocyanin biosynthesis in Malus crabapple leaves. However, little is known about how the promoter regulates $M c M Y B 10$ expression and influences the substantial variation in leaf anthocyanin accumulation and coloration that is observed in different crabapple cultivars. In this study, we analyzed leaf coloration, anthocyanin levels, and the expression levels of $M c M Y B 10$ in the leaves of 15 crabapple cultivars with three leaf colors at various development stages, and showed that the expression of McMYB10 correlates positively with anthocyanin accumulation. We also examined the relationship between the number of $\mathrm{R} 6$ and $\mathrm{R} 1$ elements in the McMYB10 promoters of the different cultivars and the pigmentation of the new buds of spring-red cultivars, as well as the methylation level of the $M c M Y B 10$ promoters at different development stages in three representative crabapple cultivars. The ratio of $\mathrm{R} 6 / \mathrm{R} 1$ minisatellites in the promoters correlated with the color and anthocyanin accumulation in new crabapple buds, and we concluded that the differences in promoter structure and methylation level of the McMYB10 promoters coordinately affect the leaf color of crabapple cultivars.

Anthocyanins are a class of pigments that contribute to the red, blue, and purple colors of many organs and tissues in a wide range of plant species (Field et al., 2001; Honda and Saito, 2002). Anthocyanins also have diverse other functions, including providing protection against abiotic stresses, particularly ultraviolet irradiation, and biotic factors, such as phytophagous insects, as well as attracting pollinators (Misra et al., 2010; Page et al., 2012). Moreover, as they have antioxidant properties that can help prevent cancer, as well as cardiovascular and neurodegenerative diseases, they are considered beneficial to human health (Boudet, 2007; Martin, 2013).

Many aspects of the molecular genetics and biochemistry of the anthocyanin biosynthetic pathway have been well characterized. For example, the genes in encoding chalcone synthase, chalcone isomerase, flavanone 3hydroxylase, flavonoid 3' -monooxygenase, dihydroflavonol 4-reductase, anthocyanidin synthase, uridine diphosphate glucose-flavonoid 3-o-glucosyltransferase, flavonol synthase, leucoanthocyanidin reductase, and anthocyanidin reductase, which are all components of the biosynthetic pathway, have been characterized in several plant species, including Arabidopsis thaliana (Winkel-Shirley, 2001), Petunia hybrida (Beld et al., 1989), and Malus crabapple (Shen et al., 2012; Tian et al., 2011), as well as in species that bear fleshy fruits, such as apple (Malus domestica) (Chagné et al., 2013) and grape (Vitis vinifera) (Cavallini et al., 2014).

Other studies have shown that anthocyanin biosynthetic genes are regulated by a transcriptional activation complex consisting of R2R3-MYB and bHLH TFs, as well as WD40 family proteins (Bai et al., 2011; Hichri et al., 2011; Zhao et al., 2013). The MYB TF family, which is one of the largest plant TF families, has been reported to play a particularly important regulatory role. For example, the anthocyanin pathway in apple is controlled by the MYB TFs, MdMYB1, $M d M Y B 10$, and $M d M Y B A$, which are allelic to each other. The protein sequence of $M Y B A$ is $100 \%$ and $98 \%$ identical to $M Y B 1$ and $M Y B 10$, respectively, and functional assays have shown that these genes are key regulators of anthocyanin accumulation and fruit coloration (Ban et al., 2007; Espley et al., 2007; Takos et al., 2006). A recent study also showed that MYB110a, a paralog of MYB10, is associated with a red-flesh cortex phenotype in apple, and that MYB10 and MYB110a have conserved functions in some cultivars, but differ in their expression patterns during fruit maturity (Chagné et al., 2013). The pear (Pyrus pyrifolia), peach (Prunus persica), and mangosteen (Garcinia mangostana L.) MYB10 homologs have also been shown to regulate fruit anthocyanin biosynthesis (Feng et al., 2010; Palapol et al., 2009; Ravaglia et al., 2013). In addition, we previously identified MYB TF, named McMYB10, which is expressed in the petals and leaves of Malus crabapples, and that has the same function as $M d M Y B 10, M d M Y B A$, and MdMYB1 (Jiang et al., 2014; Tian et al., 2015). Genetic transformation and DNA-binding assays demonstrated that the McMYB10 TF functions to promote anthocyanin accumulation by regulating the expression of the crabapple $M c F 3^{\prime} H$ gene in the flavonoid pathway, thereby promoting red leaf and petal coloration (Tian et al., 2015).

Subsequent analysis showed that a rearrangement of the 23-base pair (bp) sequences in the promoter region of $M d M Y B 10$ was responsible for the difference in gene expression between the white- and red-flesh apples (Espley et al., 2009). Specifically, the R1: MdMYB10 promoter has a single MdMYB10 binding motif, and is only present in whitefleshed apples, whereas the R6:MdMYB10 promoter, which is present in red-fleshed apples, has five additional tandem repeats of the MdMYB10 binding motif. This allelic rearrangement in the promoter of MYB10 has generated an autoregulatory locus, and this autoregulation mechanism is sufficient to account for the increase in MYB10 transcript levels and subsequent ectopic accumulation of anthocyanins throughout the plant (Espley et al., 2009). In a previous study, we 
found the same structural difference in the McMYB10 promoters, where six repeated minisatellites and a dinucleotide microsatellite were found in the promoter region of the an ever-red leaf crabapple cultivar, but only one minisatellite and a dinucleotide microsatellite were present in an evergreen leaf cultivar (Tian et al., 2015).

Leaf color is a key determinant of the commercial value of many ornamental plant species, and Malus crabapples varieties show a diverse range of leaf, flower, and fruit and leaf colors. There are three kinds of Malus crabapples leaf-color cultivars, including the "ever-red-leaf," the "ever-green-leaf," and the "spring-red-leaf" cultivars (Tian et al., 2011; Tai et al., 2014), and so crabapple ornamental apple germplasm is a valuable model for studying plant pigmentation mechanisms. We reported that R6 and R1: McMYB10 promoters are present in the ever-red and ever-green-leaf cultivars, respectively (Tian et al., 2015). However, the promoter structures of McMYB10 in cultivars with red leaves in the spring (spring-red-leaf), and the relationship between the number of R6 and R1 minisatellites in the promoter and anthocyanin accumulation in the leaves, are still unknown.

To investigate the role of the McMYB10 promoter in leaf color formation, we analyzed its structure and distribution in three cultivars with different leaf colors. Specifically, we examined the relationship between the number of R6 and R1 elements in the McMYB10 promoters and the pigmentation of new buds in cultivars that have red leaves in the spring. We also examined the promoter methylation at different development stages to investigate its effects on anthocyanin accumulation and McMYB10 expression. We describe here difference in promoter structure and minisatellite copy

Received for publication 9 Nov. 2016. Accepted for publication 3 Jan. 2017.

Financial support was provided by the National Natural Science Foundation of China (31501723), the Project of Construction of Innovative Teams and the Teacher Career Development for Universities and Colleges Under Beijing Municipality (IDHT20150503), Beijing Technology Innovation Service Capacity Construction-Research Plan (KM201610020003), and the Beijing Personnel of High Caliber Cultivation Project (2014000020124G076).

We thank the Fruit Tree Key Laboratory at the Beijing University of Agriculture. We also thank the Beijing Key Laboratory for Agricultural Application and New Technique and Beijing Nursery Engineering Research Center for Fruit Crops for providing experimental resources. We are grateful to all technicians in the BUA Crabapple Germplasm Resource Garden. We thank PlantScribe (www.plantscribe.com) for carefully editing this manuscript.

The authors declare that the research was conducted in the absence of any commercial or financial relationships that could be construed as a potential conflict of interest.

${ }^{1}$ These authors contributed equally to this work. ${ }^{2}$ Corresponding author. E-mail: yaoyc_20@126.com. number, as well as the methylation level of the McMYB10 promoters, in the context of crabapple leaf coloration.

\section{Materials and Methods}

Plant materials and growth conditions. Five-year-old trees of the ever-red-leaf cultivar Malus cv. Royalty; cultivars with leaves that are red in the spring (springred; 'Radiant', 'Indian Magic', 'Prairifire', 'Red Begonia', 'Red Splender', 'Kelsy', and 'Strawberry Jelly'); and the evergreen-leaf cultivars Spring Snow, Donald,
Dolgo, Snowdrift, Red Jade, Flame, and Jewelberry grafted on Malus hupehensis were planted in the Crabapple Germplasm Resources Nursery in the Beijing University of Agriculture. We selected five trees of each 15 cultivars that showed similar growth and collected leaf samples from annual branches growing in all four compass directions on the outer edge of each canopy as five biological replicates. The average weight of each leaf at $6 \mathrm{~d}$ was $0.1-0.2 \mathrm{~g}$ and at $30 \mathrm{~d}$ was $0.8-1.0 \mathrm{~g}$. Thirty leaves were collected in each tree of 15 cultivars and at each of the five different
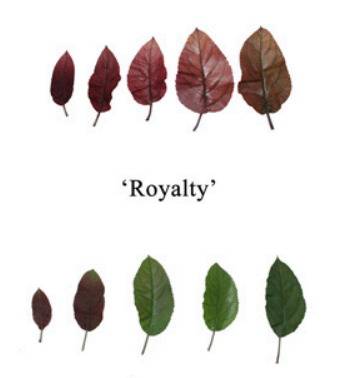

'Prairifire'

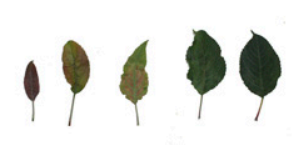

'Kelsy'

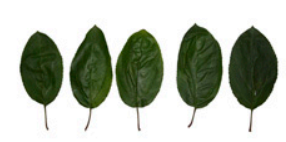

'Donald'

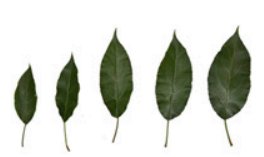

'Red Jade'

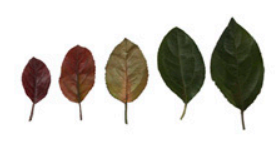

'Radiant'

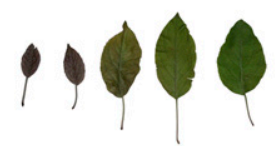

'Red Begonia'

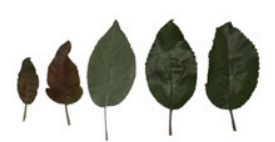

'Strawberry Jelly'

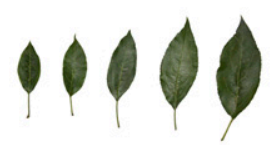

'Dolgo'

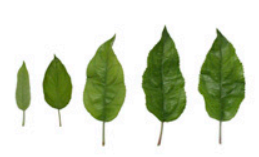

'Flame'

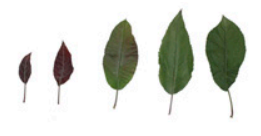

'Indian Magic'

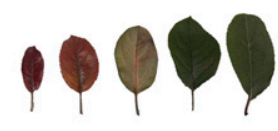

'Red Splender'

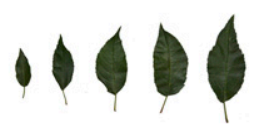

'Spring Snow'

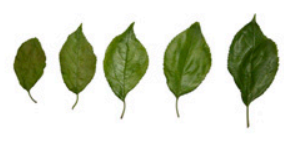

'Snowdrift'

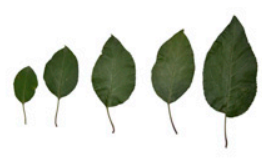

'Jewelberry'

Fig. 1. Five leaf developmental stages with different colors used for the analyses. Malus cv. Royalty is an ever-red-leaf cultivar; 'Radiant', 'Indian Magic', 'Prairifire', 'Red Begonia', 'Red Splender', 'Kelsy', and 'Strawberry Jelly' are spring-red-leaf cultivars; 'Spring Snow', 'Donald', 'Dolgo', 'Snowdrift', 'Red Jade', 'Flame', and 'Jewelberry' are ever-green-leaf cultivars. Scale bars $=1 \mathrm{~cm}$.

Table 1. Primer sequences used in this study.

\begin{tabular}{|c|c|c|c|}
\hline $\begin{array}{l}\text { Accession } \\
\text { number }\end{array}$ & ID & Sequence $\left(5^{\prime}-3^{\prime}\right)$ & Analytical use \\
\hline \multirow[t]{2}{*}{$\overline{\text { EF528482 }}$} & APX-F & ATCAAGCAACAGTTCCCC & Copy number \\
\hline & APX-R & GGGGAACTGTTGCTTGAT & qRT-PCR \\
\hline \multirow[t]{2}{*}{ AB557643 } & $\mathrm{R} 6+\mathrm{R} 1-\mathrm{F}$ & GCATTGCCTCTTCATCTCTCTAC & Copy number \\
\hline & $\mathrm{R} 6+\mathrm{R} 1-\mathrm{R}$ & CAGCAAACACCCAAАATCCСТ & qRT-PCR \\
\hline \multirow[t]{2}{*}{ AB557643 } & R6-F & CTGGTTAGACTGGTAGCT & Copy number \\
\hline & R6-R & AGACTCCAGAAAGACACC & qRT-PCR \\
\hline \multirow[t]{2}{*}{ JX162681 } & MYB10M-F & GTTAGTTTGTAATAGATTGAGATAGGT & Methylation \\
\hline & MYB10-R & AACAATTAAATTTCAAATAAAAAACTAC & analysis \\
\hline \multirow[t]{2}{*}{ AB557643 } & PMYB10-F & CTTATGGTGGTCAAAGATGTGTGTT & Promoter \\
\hline & PMYB10-R & CCACAGAAGCAAACACTGACAAG & cloning \\
\hline \multirow[t]{2}{*}{ JX162681 } & MYB10-F & GGCGCATGATCTTGGCGACAGT & qRT-PCR \\
\hline & MYB10-R & ACGCCACCACAAACGTCGTCG & \\
\hline \multirow{2}{*}{ AB638619 } & MdActin-F & GCTCCCGAAGAACACCCAG & qRT-PCR \\
\hline & MdActin-R & GGACCCTCCAATCCAGACA & \\
\hline
\end{tabular}

qRT-PCR = quantitative real-time reverse transcription polymerase chain reaction. 
developmental stages (stage 1, $3 \mathrm{~d}$ after budding; stage 2, $9 \mathrm{~d}$ after budding; stage 3, $15 \mathrm{~d}$ after budding; stage 4, $21 \mathrm{~d}$ after budding; stage 5, $30 \mathrm{~d}$ after budding) (Fig. 1). Of each leaf samples, 2 and $1 \mathrm{~g}$ were used for RNA extraction and high-pressure liquid chromatography (HPLC) analysis, respectively. At stage $1,1 \mathrm{~g}$ of leaf samples was used for DNA extraction.

HPLC analysis. Crabapple leaves $(\approx 0.8-$ $1.0 \mathrm{~g}$ fresh weight) were extracted with $10 \mathrm{~mL}$ extraction solution (methanol:water:formic acid:trifluoroacetic acid $=70: 27: 2: 1$ ) as previously described (Tian et al., 2015) at $4{ }^{\circ} \mathrm{C}$ in the dark for $72 \mathrm{~h}$, with shaking every $6 \mathrm{~h}$. Each sample was filtered through sheets of qualitative filter paper and the filtrates were then passed through $0.22-\mu \mathrm{m}$ reinforced nylon membrane filters (Billerica, MA). The samples were then analyzed by HPLC on an HPLC1100-DAD system (Agilent Technologies, Waldbronn, Germany), using trifluoroacetic acid formic acid:water (0.1:2:97.9) as mobile phase A, and trifluoroacetic acid:formic acid:acetonitrile:water (0.1:2:48:49.9) as mobile phase B. The gradients used were as follows: $0 \mathrm{~min}$, $30 \% \mathrm{~B} ; 10 \mathrm{~min}, 40 \% \mathrm{~B} ; 50 \mathrm{~min}, 55 \% \mathrm{~B} ; 70 \mathrm{~min}$, $60 \% \mathrm{~B}$; and $80 \mathrm{~min}, 30 \% \mathrm{~B}$ and detection was performed at $520 \mathrm{~nm}$ for anthocyanins and $350 \mathrm{~nm}$ for flavonols (Revilla and Ryan, 2000) (Supplemental Fig. 1). All samples were analyzed in five biological replicates.

Cloning and sequence analysis of the $M c M Y B 10$ promoter region. To analyze the differences in McMYB10 promoter sequences between the different cultivars, genomic DNA was isolated from leaves using the Plant Genomic DNA Kit (TIANGEN Biotech Co., Ltd, Beijing, China). Cloning primers PMYB10-F and PMYB10-R were designed as previously described (Tian et al., 2015) (Table 1). Polymerase chain reaction (PCR) products were cloned into the pMD-19T vector and sequenced.

Quantitative real-time $P C R$ analysis. Total RNA was extracted from crabapple leaves using an RNA Extraction Kit (Aidlab, Beijing, China) according to the manufacturer's instructions. DNase I (TaKaRa, Ohtsu, Japan) was added to remove genomic DNA, and the samples were then subjected to cDNA

Table 2. High-pressure liquid chromatography (HPLC) analysis of anthocyanin (cyanidin) levels in the leaves of 15 crabapple cultivars.

\begin{tabular}{|c|c|c|c|c|c|c|}
\hline Leaf phenotypes & Cultivars/phyllotaxis & $1\left(\mu \mathrm{g} \cdot \mathrm{g}^{-1}\right)$ & $2\left(\mu \mathrm{g} \cdot \mathrm{g}^{-1}\right)$ & $3\left(\mu \mathrm{g} \cdot \mathrm{g}^{-1}\right)$ & $4\left(\mu \mathrm{g} \cdot \mathrm{g}^{-1}\right)$ & $5\left(\mu \mathrm{g} \cdot \mathrm{g}^{-1}\right)$ \\
\hline Ever-red cultivar & Royalty & $623.36 \pm 6.56 \mathrm{aA}$ & $702.60 \pm 17.14 \mathrm{aA}$ & $710.88 \pm 27.13 \mathrm{aA}$ & $231.45 \pm 3.47 \mathrm{bA}$ & $16.31 \pm 0.98 \mathrm{cG}$ \\
\hline & Indian Magic & $282.57 \pm 0.35 \mathrm{aC}$ & $235.70 \pm 0.88 \mathrm{aD}$ & $39.20 \pm 0.79 \mathrm{bD}$ & $26.05 \pm 0.12 \mathrm{cF}$ & $19.39 \pm 0.59 \mathrm{dF}$ \\
\hline & Prairifire & $434.46 \pm 1.01 \mathrm{aB}$ & $51.70 \pm 0.62 \mathrm{dF}$ & $64.25 \pm 0.24 \mathrm{bC}$ & $53.61 \pm 0.08 \mathrm{cC}$ & $45.24 \pm 0.77 \mathrm{eB}$ \\
\hline & Red Splender & $155.20 \pm 2.18 \mathrm{aD}$ & $85.35 \pm 0.72 \mathrm{bE}$ & $70.95 \pm 1$. & $78.91 \pm 0.38 \mathrm{cB}$ & $55.30 \pm 0.73 \mathrm{eA}$ \\
\hline & Kelsy & $151.59 \pm 22.19 \mathrm{bD}$ & $111.05 \pm 9.02 \mathrm{aB}$ & $58.11 \pm 3.04 \mathrm{cC}$ & $29.62 \pm 0.11 \mathrm{dE}$ & $28.53 \pm 0.78 \mathrm{dD}$ \\
\hline & Strawberry Jelly & $55.02 \pm 0.52 \mathrm{aE}$ & $51.94 \pm 0.86 \mathrm{aG}$ & $30.94 \pm 0.03 \mathrm{aD}$ & $28.53 \pm 0.78 \mathrm{bE}$ & $25.44 \pm 0.91 \mathrm{cE}$ \\
\hline
\end{tabular}

1, $3 \mathrm{~d}$ after budding; 2, $9 \mathrm{~d}$ after budding; 3, $15 \mathrm{~d}$ after budding; 4, $21 \mathrm{~d}$ after budding; 5, 30 d after budding. Error bars indicate the mean $\pm \mathrm{SE}$ of five replicate reactions. Small letters and capital letters (in column) indicate significance at $P<0.05$ and $P<0.01$, respectively, according to Duncan's multiple range test.

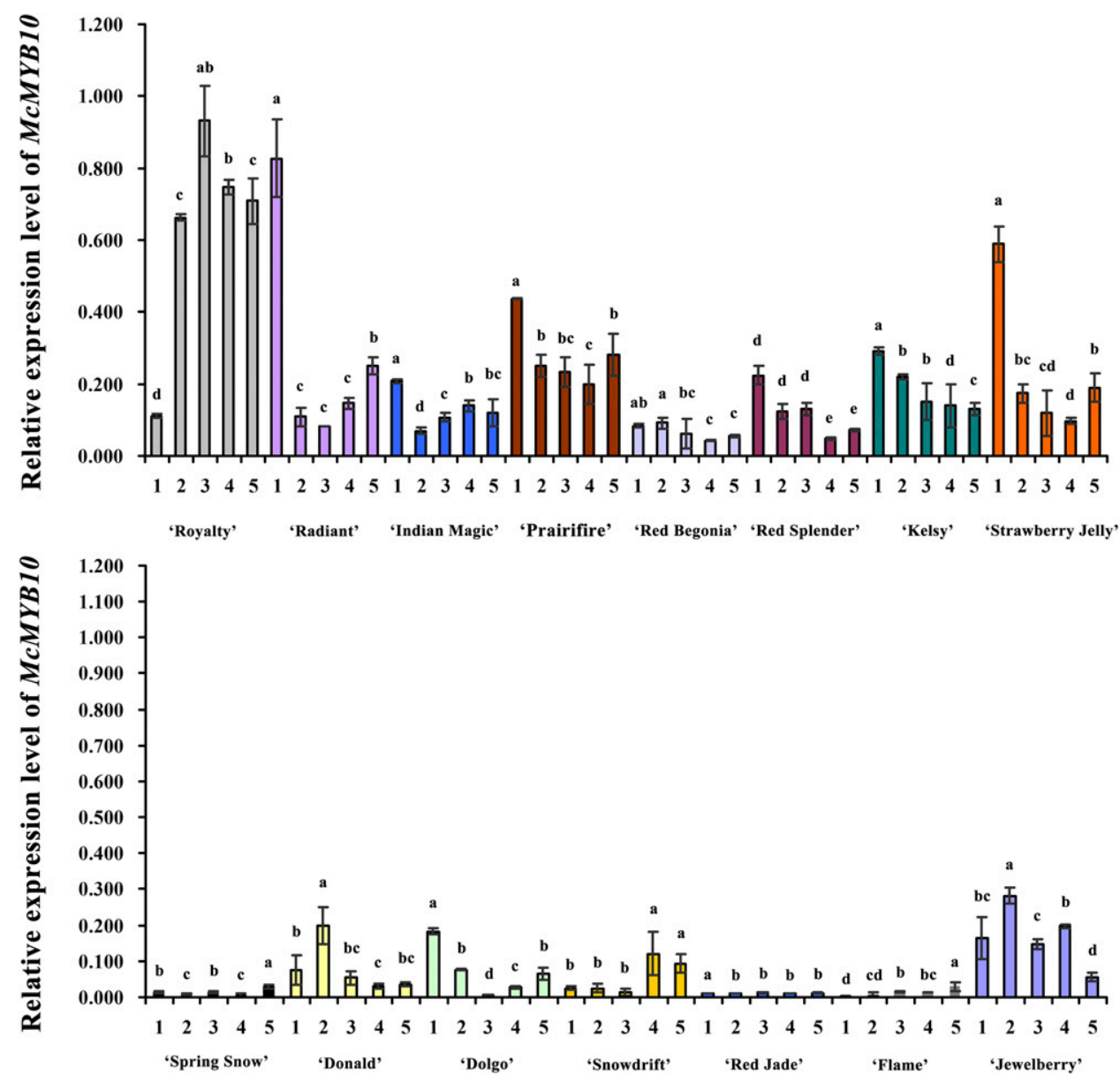

Fig. 2. Expression profiles of Malus crabapple McMYB10 during five leaf developmental stages. Real-time polymerase chain reaction was used to analyze McMYB10 expression patterns in the leaves of 15 crabapple cultivars (names on the $x$ axis). The $18 S$ gene was used as the reference gene. 1 to 5 represent leaf developmental stages 1 to 5 . Error bars on each bar indicate the mean \pm SE of three replicate reactions. 
synthesis using the Access RT-PCR System (Promega, Madison, WI), according to the manufacturer's instructions. The expression levels of the McMYB10 genes were analyzed using quantitative real-time PCR (qRT-PCR) on a CFX96 Real-Time PCR System (BIO-RAD), according to the manufacturers' instructions. The total reaction volume was $20 \mu \mathrm{L}$, comprising $9 \mu \mathrm{L}$ of $2 \times$ SYBR Green qPCR Mix (TaKaRa), $0.1 \mu \mathrm{M}$ specific primers (each), and $100 \mathrm{ng}$ of template cDNA. The reaction mixtures were heated to $95^{\circ} \mathrm{C}$ for $30 \mathrm{~s}$, followed by 39 cycles at $95^{\circ} \mathrm{C}$ for $10 \mathrm{~s}, 55^{\circ} \mathrm{C}$ for $15 \mathrm{~s}$, and $72{ }^{\circ} \mathrm{C}$ for $30 \mathrm{~s}$. A melting curve was generated for each sample at the end of each run to ensure the purity of the amplified products. The transcript levels were normalized using the Malus $18 \mathrm{~S}$ ribosomal RNA gene (DQ341382) as the internal controls and calculated using the $2^{-\triangle \Delta \mathrm{Ct}}$ analysis method (Livak and Schmittgen, 2001). The gene-specific PCR primers were designed using NCBI Primer BLAST (https:// www.ncbi.nlm.nih.gov/tools/primer-blast/) and are listed in Table 1.

Measurement of methylation level. Bisulfite-sequencing PCR (BSP) analysis was performed as described in Telias et al. (2011). Briefly, genomic DNA $(1.0 \mu \mathrm{g})$ was subjected to bisulfite treatment and purification using the DNA Bisulfite Conversion Kit (TIANGEN Biotech). Digested DNA was included in PCR reactions using the methylation-specific PCR kit (TIANGEN Biotech) following the manufacturer's instructions. The PCR products were purified and cloned directly into the pMD-19 vector (Takara) for sequencing. The individual clones were sequenced 8-10 times, and the methylation level of each fragment was calculated. Primer sequences are listed in Table 1.
Calculation of copy number and statistical analysis. The dissolution curves and amplification curve of R6, R1, R6 + R1, and the reference gene, $A P X$ (EF528482), were calculated from the data obtained from qRT-PCR analysis of 15 Malus crabapple cultivars to establish the amplification efficiency. RT-qPCR analysis was carried out as described above. To determine the R6 and R1 copy numbers, a standard curve was drawn with the log starting values on the $x$ axis and cycle threshold (CT) value on the $y$ axis, using the calculation of the copy number and statistical analysis (Mason et al., 2002).

\section{Results}

Anthocyanin contents of the leaves from three types of crabapple leaf-color cultivars. Ever-red crabapple cultivar Royalty; springred cultivars (Radiant, Indian Magic, Prairifire, Red Begonia, Red Splender, Kelsy, and Strawberry Jelly); and the ever-green-leaf cultivars (Spring Snow, Donald, Dolgo, Snowdrift, Red Jade, Flame, and Jewelberry), which belong to three types of leaf-color cultivars, were used in our research (Fig. 1). To examine the variation in leaf color of the different cultivars, HPLC analysis was performed to measure the anthocyanin contents in developmental stages 1-5 of the Malus crabapple leaf-growing season. We observed that cyanidin was present at much higher level in the leaves of the ever-red cultivar Royalty than in the leaves of spring-red cultivars. 'Royalty' showed high levels of cyanidin at the first leaf developmental stage and the highest amount at stage 3, whereas the levels peaked in spring-red cultivars at stage 1 and then decreased during leaf devel- opment. In contrast, cyanidin was almost undetectable in the leaves of the ever-green cultivars (Table 2). Overall, anthocyanin concentration is correlated well with the color variation in the leaves of the different cultivars.

Expression level of McMYB10 in the leaves of different cultivars. The transcript levels of McMYB10 were determined by RT-PCR, using gene-specific primers. Expression increased gradually during leaf development in ever-red cultivar Royalty, and was significantly higher than that in the evergreen cultivars. Moreover, expression was higher at the early stages and then declined with leaf development in the spring-red cultivars, where it was typically higher than in the ever-green 'Flame' leaves at the same stages (Fig. 2). The expression levels of McMYB10 in different crabapple cultivars generally followed the patterns of anthocyanin accumulation, further suggesting that this gene plays an important role in anthocyanin accumulation in crabapple leaves.

McMYB10 promoter structure. Previous studies showed that a rearrangement in the upstream regulatory region of MYB10 is responsible for the red foliage and red fruit flesh in apple and crabapple (Espley et al., 2009; Tian et al., 2015). We therefore sequenced the minisatellite region of the McMYB10 promoter, which has been associated with anthocyanin accumulation, from 15 crabapple varieties with diverse leaf phenotypes. This revealed the presence of six repeated minisatellites (GTTAGACTGG TAGCTATTAACAA) and a dinucleotide microsatellite (GTGT) (R6 promoter) in the ever-red crabapple cultivar, but only one minisatellite and a dinucleotide microsatellite (R1 promoter) in the ever-green crabapple

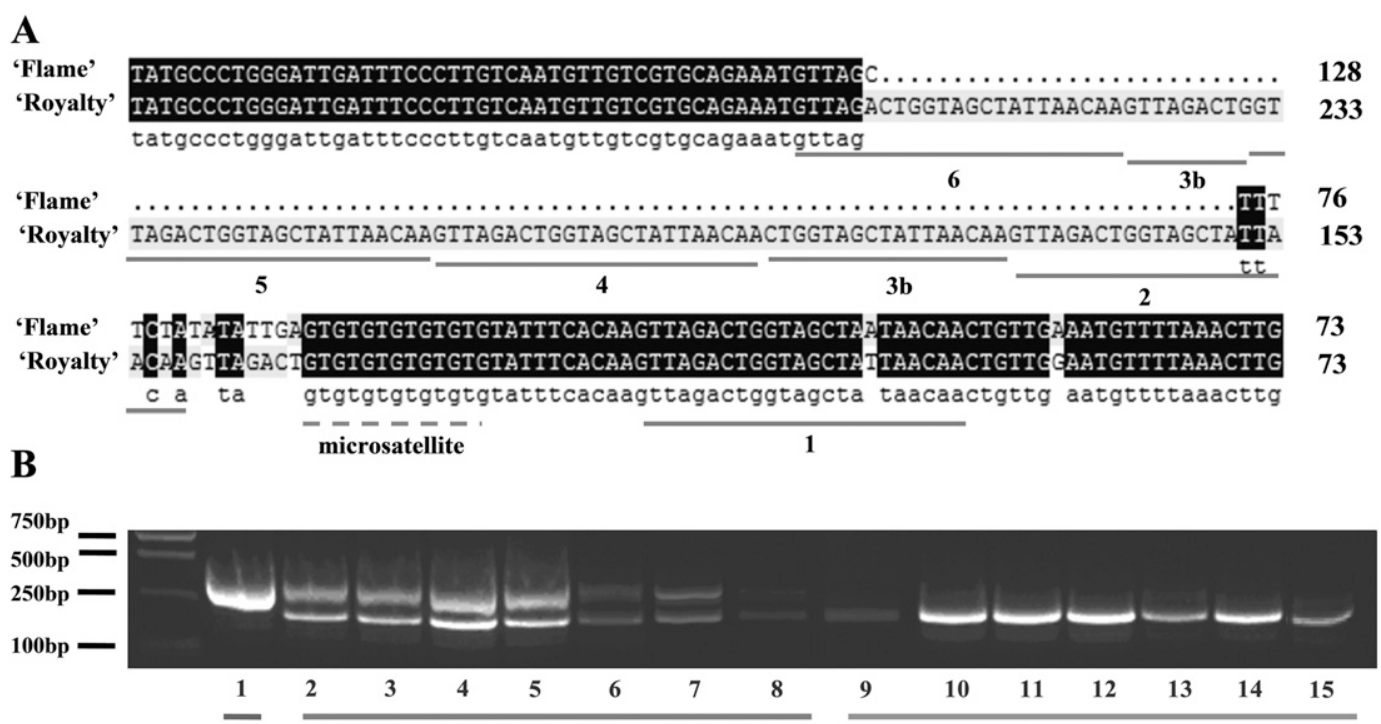

Ever-red cultivar

Spring-red cultivars

Ever-green cultivars

Fig. 3. Sequence and promoter analysis of the McMYB10 in 15 crabapple cultivars. (A) Sequence alignment of McMYB10 promoter regions of the ever-red leaf Royalty and ever-green leaf Flame crabapple cultivars. Numbers and underlined regions represent the positions of the repeat units (GTTAGACTGGTAG CTATTAACAA); the dotted line indicates microsatellite regions. (B) Polymerase chain reaction amplification of the MYB10 promoter region resulted in two fragments: a 496-base pair (bp) fragment corresponding to R6 that is present only in the red-leaf cultivar (lane 1) and a 392-bp fragment corresponding to R1 that is present only in green-leaf cultivars (lanes 9 to 15), whereas R6 and R1 are both present in spring-red-leaf cultivars (lanes 2 to 8). 
cultivars (Fig. 3A). Interestingly, the R6 and R1 promoters were both present in the spring-red crabapple cultivars, whereas the R6 or R1 promoter sequences were specific for the everred- or ever-green-leaf cultivars, respectively. The results indicate that the presence of both R1 and R6 promoter in spring-red cultivars have allowed the leaves show both red and green color during the developmental stages (Fig. 3B). These genetic polymorphisms were consistent with the leaf phenotypes.

Number of R6 and R1 minisatellites in the new buds of spring-red cultivars. Since the R6 promoter sequence determined the red leaf color, we investigated whether a difference in the number of R6 minisatellites might also explain the differences in the red color and anthocyanin content in new buds compared among different spring-red cultivars, by analyzing the promoter sequences of the 15 crabapple cultivars using qRT-PCR. We could only detect either the R6 or the R1 promoter sequences in the ever-red and ever-green-leaf cultivars, respectively. In contrast, the R6 and R1 promoters were both present in the spring-red cultivars, but in different ratios (Table 3; Supplemental Fig. 2). To further confirm the relationship between the number of R6 and $\mathrm{R} 1$ elements and new bud color and anthocyanin content, we calculated the ratio of $\mathrm{R} 6 / \mathrm{R} 1$ and the flavonoid content as a ratio of anthocyanins/flavonols (Table 4). We observed that $\mathrm{R} 6 / \mathrm{R} 1$ ratio had a similar variation trend as the anthocyanins/flavonols ratios at leaf development stage 1 , with a correlation coefficient of 0.953 (Fig. 4). This result was consistent with the R6/R1 ratio determining the color and anthocyanin accumulation in new crabapple buds.

Methylation level of the McMYB10 promoter is associated with green leaf color in spring-red cultivars. Several studies have shown that methylation may play a role in the regulation of MYB10 expression (Telias et al., 2011; Wang et al., 2013), and a CpG island has been identified in the McMYB10 promoter. To investigate whether methylation affects the anthocyanin accumulation in crabapple leaves, we measured the methylation level of the McMYB10 promoter in three typical crabapple cultivars: the ever-red cultivar Royalty, the ever-green cultivar Flame, and the spring-red cultivar Radiant. We analyzed the methylation level of cytosine in the McMYB10 promoter by BSP in the -1288 to -1595 bp region. The methylation level of the promoter gradually decreased during leaf development in 'Royalty' and 'Flame', but increased during development stages 1-3 and decreased in the last two developmental stages in 'Radiant'. Analysis of correlation coefficient indicated a clear negative relationship between the methylation level and the expression level of McMYB10 (Fig. 5). These data suggested that the methylation level of the McMYB10 promoter in crabapple leaves can affect gene expression, and that differences in R6 and $\mathrm{R} 1$ copy numbers, together with variation in degree of methylation of the McMYB10

Table 3. Number of R6 and R1 promoter elements in 15 crabapple cultivars.

\begin{tabular}{llcc}
\hline Leaf phenotypes & \multicolumn{1}{c}{ Cultivars } & Number of R6 elements & Number of R1 elements \\
\hline Ever-red cultivar & Royalty & 7 & 0 \\
Spring-red cultivars & Radiant & 5 & 3 \\
& Indian Magic & 4 & 4 \\
& Prairifire & 3 & 1 \\
& Red Begonia & 3 & 3 \\
& Red Splender & 2 & 4 \\
& Kelsy & 11 & 4 \\
Ever-green cultivar & Strawberry Jelly & 7 & 2 \\
& Spring Snow & 0 & 4 \\
& Donald & 0 & 10 \\
& Dolgo & 0 & 14 \\
& Snowdrift & 0 & 10 \\
& Red Jade & 0 & 7 \\
& Flame & 0 & 5 \\
\hline
\end{tabular}

Table 4. The content of anthocyanins and flavonols, as well as the number of R6 and R1 promoter elements, in 15 crabapple cultivars.

\begin{tabular}{lcrcc}
\hline Cultivars & $\begin{array}{c}\text { Cyanidin-3-o-glucoside } \\
\left(\mu \mathrm{g} \cdot \mathrm{g}^{-1}\right)\end{array}$ & $\begin{array}{c}\text { Flavonol } \\
\left(\mu \mathrm{g} \cdot \mathrm{g}^{-1}\right)\end{array}$ & $\begin{array}{c}\text { Number of } \\
\text { R6 elements }\end{array}$ & $\begin{array}{c}\text { Number of } \\
\text { R1 elements }\end{array}$ \\
\hline Radiant & $398.19 \pm 3.66$ & $1,192.26 \pm 7.12$ & 5 & 3 \\
Indian Magic & $282.57 \pm 0.35$ & $918.9 \pm 5.36$ & 4 & 4 \\
Prairifire & $434.46 \pm 1.01$ & $876.34 \pm 4.59$ & 3 & 1 \\
Red Begonia & $153.33 \pm 0.78$ & $526.43 \pm 3.71$ & 3 & 3 \\
Red Splender & $155.20 \pm 2.18$ & $1,349.31 \pm 3.56$ & 2 & 4 \\
Kelsy & $151.59 \pm 22.19$ & $262.41 \pm 5.66$ & 11 & 4 \\
Strawberry Jelly & $55.02 \pm 0.52$ & $93 \pm 3.16$ & 7 & 2 \\
\hline
\end{tabular}
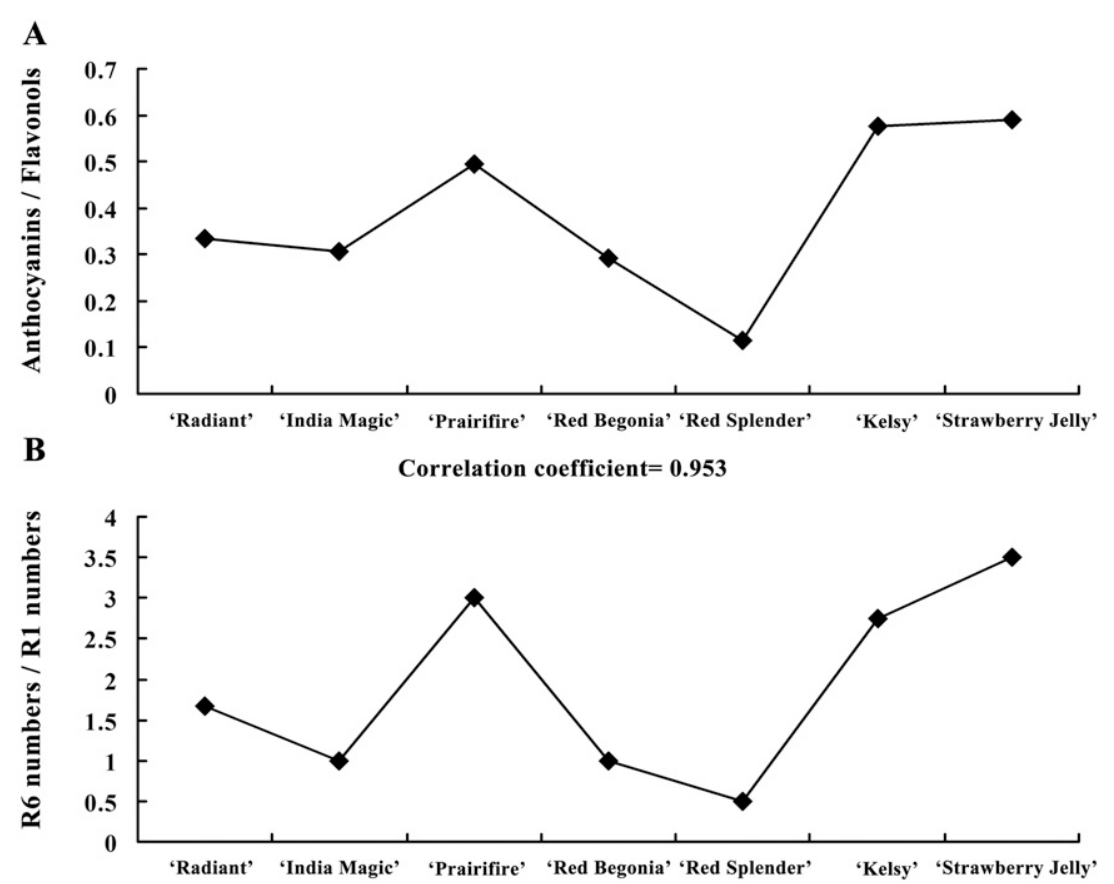

Fig. 4. The ratio of R6/R1 correlates positively with the content of anthocyanin/flavonol in leaf developmental stage 1 in spring-red-leaf cultivars. (A) The variation in the anthocyanin/flavonol content ratio in leaf developmental stage 1 in spring-red-leaf cultivars. (B) The variation in the R6/R1 ratio in leaf development stage 1 in spring-red-leaf cultivars.

promoter, coordinately regulate color change in spring-red cultivars.

\section{Discussion}

Anthocyanins are a class of secondary metabolites that contribute to the red, blue, and purple colors of a range of leaves, flowers, and fruits. Previous studies have shown how a rapid red coloration response to light and low temperature is likely to be caused by an elevation in the expression of $M Y B 10$ genes, driving anthocyanin biosynthesis gene expression and, consequently, anthocyanin pigmentation in Malus plants (Ban et al., 2007; Tian et al., 2015). The $M Y B 10$ gene is allelic to a peel-expressed gene, MdMYB1 or MYBA (Ban et al., 2007; 

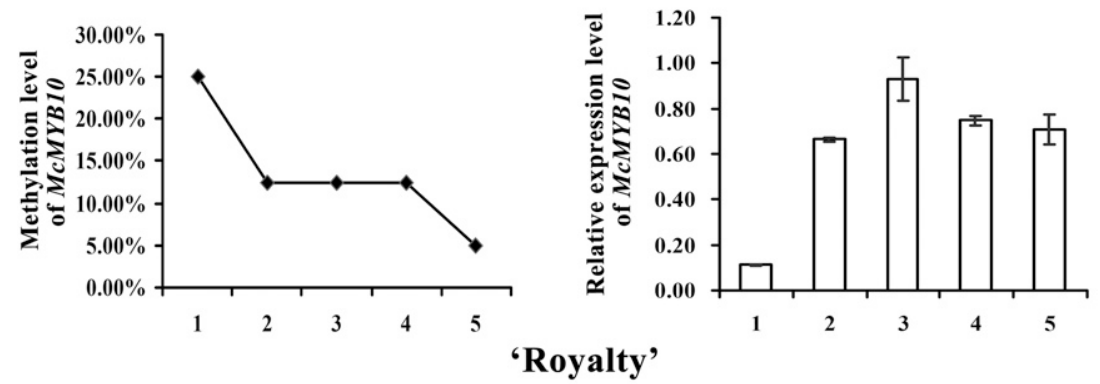

B

Correlation coefficient $=\mathbf{- 0 . 7 1 5 9}$
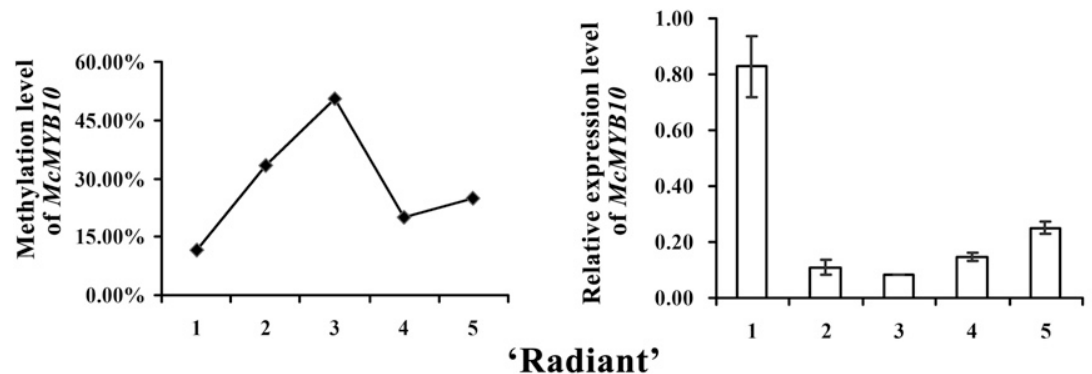

C

Correlation coefficient $=\mathbf{- 0 . 8 1 0 8}$

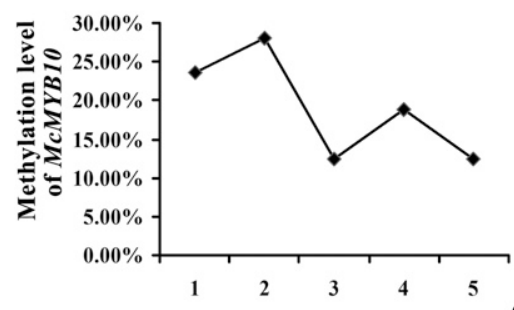

'Flame'
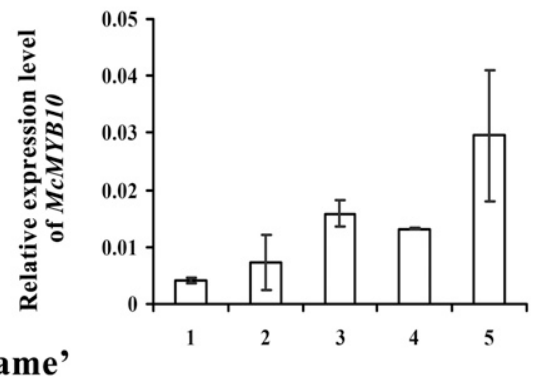

Fig. 5. Correlation between the methylation and expression level of $M c M Y B 10$ in three representative cultivars with different leaf colors. (A) The methylation level of the McMYB10 promoter and the expression level of McMYB10 in 'Royalty'. (B) The methylation level of the McMYB10 promoter and the expression level of McMYB10 in 'Radiant'. (C) The methylation level of the McMYB10 promoter and the expression level of McMYB10 in 'Flame'.

Lin-Wang et al., 2010; Takos et al., 2006), and genetic studies suggests that these alleles are the major controllers of apple peel (Ban et al., 2007; Zhu et al., 2011) and flesh and foliage color (Espley et al., 2007). MdMYB1/ $M Y B A$ expression is induced by light and cold temperature, whereas the MYB10 transcript levels are suppressed at higher temperatures (Ban et al., 2007; Lin-Wang et al., 2010; Takos et al., 2006). McMYB10 play an important role in leaf and petal coloration, by promoting the expression of downstream genes in crabapple (Jiang et al., 2014; Tian et al., 2015). When MYB10 sequences from diverse apple cultivars were sequenced, no differences were found in the encoded protein sequences; however, differences in the expression of MYB10 between apple cultivars were associated with differences in its promoter (Espley et al., 2009).

In a previous study, a 23-bp repeat motif in the upstream regulatory region of alleles of MYB10 was found to present only in red-fleshed apples and red-leaf crabapples
(Espley et al., 2009). Moreover, this promoter allele was shown to be responsible for the increased accumulation of anthocyanins, and the number of repeat units correlates with an increase in transactivation by the MYB10 protein (Espley et al., 2009). In our study, we determined the McMYB10 promoter structures of 15 crabapple cultivars, which collectively represent three types of leaf color. The ever-green-leaf cultivars tested contained only the R1 promoter element and the ever-red-leaf cultivar had only the R6 promoter element, whereas the spring-red cultivars had both the R1 and R6 promoter elements. In a previous study, red-fleshed apple cultivars were reported to have both R6 and R1, or R6 only (Espley et al., 2009); however, we conclude that in crabapple, the presence of both minisatellite types results in a spring-red phenotype (Fig. 3). Based on these results, we conclude that the absence of the minisatellite R6 missing from the ever-green cultivars is the predominant reason for their green leaf color, whereas the presence of both the R6 and $\mathrm{R} 1$ elements in the spring-red-leaf cultivars allows them to have both red and green leaves simultaneously. In addition, the ever-red-leaf cultivar Royalty, which only had an R6 element, showed a red color in its leaves, even in late developmental stages.

Extant apple chromosome homologies are derived from a putative nine-chromosome ancestor (Velasco et al., 2010). Each doublet of the eight apple chromosomes (3-11, 5-10, $9-17$, and 13-16) is principally derived from one ancestor. The MYB10 and MYB1 genes are located at very similar positions on linkage group 9, and it was concluded that these genes were allelic (Lin-Wang et al., 2010; Zhu et al., 2011). Additionally, MYB110a, a paralog of $M Y B 10$ was recently, located to chromosomes 17 (Chagné et al., 2013). These MYB TFs maybe share a common ancestor and coordinately regulated the red color formation in apple. However, the different numbers of R6 and $\mathrm{R} 1$ promoters in apple genome, and the functions of R6 and R1 promoter numbers on coloration are still unknown. PCR analysis showed that both minisatellites were present in spring-red crabapple cultivars, and that the anthocyanin levels in new buds from these cultivars were different. We speculated that the ratio of R6 and R1 satellites might determine the color of the new buds, and indeed the qRT-PCR results showed a similar trend in R6/R1 with the content of anthocyanins/ flavonols in leaf development stage 1 of different cultivars. This is consistent with the dosage of the R6 and R1 elements playing an important role in the coloration of new crabapple buds (Fig. 4). At present, R6 and R1 promoter sequences are used as molecular markers to determine the fruit color (Zhang et al., 2014) and leaf color in apple breeding. Furthermore, the ratio of R6/R1 can be used as a key evaluation factor to analyze the color of the new buds in spring-red-leaf crabapple hybrids. Interestingly, most crabapple cultivars are diploid and the ratio of R6/R1 or $\mathrm{R} 1 / \mathrm{R} 6$ is not duple 2, and there may be copy number variation of the two promoters in specific cultivars as a consequence of longterm evolution and hybridization.

DNA methylation is a critical feature of epigenetic regulation in many eukaryotes (Wang et al., 2013). Several studies have shown that the methylation pattern of the MYB10 promoter affects plant color change. One example is fruit of the Honey Crisp cultivar, where a specific methylation pattern caused a striped pigmentation (Telias et al., 2011). A comparison of the methylation level in the pear (Pyrus communis) PcMYB10 promoter between the MRB (Max Red Bartlett) and MRB-G (MRB green-skinned sport) varieties revealed a correlation between methylation and the green-skin phenotype (Wang et al., 2013). We therefore predicted that methylation might also affect the expression of McMYB 10 in crabapple leaves, and indeed our results showed a significant negative relationship between DNA methylation in the McMYB 10 promoter and McMYB 10 transcript levels. In MRB and MRB-G pear fruits, and in 
red- and green-striped apple cultivars, there are no sequence differences in the MYB10 promoter sequences, while the significant methylation levels differ (Wang et al., 2013). However, significantly promoter sequence difference and methylation level variation were exist simultaneous in spring-red-leaf crabapple cultivars. We conclude that the promoter sequence and methylation level of MCMYB10 coordinately regulate the $M c M Y B 10$ expression (Fig. 5). Meanwhile, the main reason for the methylation levels in the spring-red cultivars were much higher than that in the other two leaf phenotype types of cultivars will trigger a series of future research projects to elucidate the mechanisms governing methylation levels in the spring-red cultivars.

Variation in methylation patterns has the potential to play a role in phenotypic plasticity as a response to environmental stress, and the difference in promoter sequences is mainly caused by evolutionary selection and mutation. Most of the ever-red- and springred-leaf crabapple cultivars originated from Europe and the United States, whereas most of the ever-green-leaf cultivars originated from China and other Asia countries. Differences in McMYB10 methylation levels and promoter sequences between the three leaf color types likely reflect environmental/geographic adaptions and differences in the evolutionary selection pressure; however, these have yet to be identified.

\section{Literature Cited}

Bai, Y.H., S. Pattanaik, B. Patra, R.J. Werkman, H.C. Xie, and L. Yuan. 2011. Flavonoid related basic helix-loop-helix regulators, NtAnla and $\mathrm{NtAn} 1 \mathrm{~b}$, of tobacco have originated from two ancestors and are functionally active. Planta 234:363-375.

Ban, Y., C. Honda, Y. Hatsuyama, M. Igarashi, H. Bessho, and T. Moriguchi. 2007. Isolation and functional analysis of a MYB transcription factor gene that is a key regulator for the development of red coloration in apple skin. Plant Cell Physiol. 48:958-970.

Beld, M., C. Martin, H. Huits, A.R. Stuitje, and A.G.M. Gerats. 1989. Flavonoid synthesis in Petunia hybrida: Partial characterization of dihydroflavonol-4-reductase genes. Plant Mol. Biol. 13:491-502.

Boudet, A.M. 2007. Evolution and current status of research in phenolic compounds. Phytochemistry 68:2722-2735.

Cavallini, E., S. Zenoni, L. Finezzo, F. Guzzo, A. Zamboni, L. Avesani, and G.B. Tornielli. 2014. Functional diversification of grapevine MYB5a and MYB5b in the control of flavonoid biosynthesis in a petunia anthocyanin regulatory mutant. Plant Cell Physiol. 55:517-534.

Chagné, D., K. Lin-Wang, R.V. Espley, R.K. Volz, N.M. How, S. Rouse, C. Brendolise, C.M. Carlisle, S. Kumar, N. De Silva, D. Micheletti, T. McGhie, R.N. Crowhurst, R.D. Storey, R. Velasco, R.P. Hellens, S.E. Gardiner, and A.C. Allan. 2013. An ancient duplication of apple MYB transcription factors is responsible for novel red fruit-flesh phenotypes. Plant Physiol. 161:225-239.

Espley, R.V., C. Brendolise, D. Chagne, S. KuttyAmma, S. Green, R. Volz, J. Putterill, H.J. Schouten, S.E. Gardiner, R.P. Hellens, and A.C. Allan. 2009. Multiple repeats of a promoter segment causes transcription factor autoregulation in red apples. Plant Cell 21:168-183. Espley, R.V., R.P. Hellens, J. Putterill, D.E. Stevenson, S. Kutty-Amma, and A.C. Allan. 2007. Red colouration in apple fruit is due to the activity of the MYB transcription factor, MdMYB10. Plant J. 49:414-427.

Feng, S.Q., Y.L. Wang, S. Yang, Y.T. Xu, and X.S. Chen. 2010. Anthocyanin biosynthesis in pears is regulated by a R2R3-MYB transcription factor PyMYB10. Planta 232:245255.

Field, T.S., D.W. Lee, and N.M. Holbrook. 2001. Why leaves turn red in autumn. The role of anthocyanins in senescing leaves of redosier dogwood. Plant Physiol. 127:566-574.

Hichri, L., F. Barrieu, J. Bogs, C. Kappel, S. Delrot, and V. Lauvergeat. 2011. Recent advances in the transcriptional regulation of the flavonoid biosynthetic pathway. J. Expt. Bot. 62:2465-2483.

Honda, T. and N. Saito. 2002. Recent progress in the chemistry of polyacylated antho-cyanins as flower color pigment. Heterocycles 56:633-692.

Jiang, R., J. Tian, T.T. Song, J. Zhang, and Y.C. Yao. 2014. The Malus crabapple transcription factor McMYB10 regulates anthocyanin biosynthesis during petal coloration. Sci. Hort. 166:42-49.

Lin-Wang, K., K. Bolitho, K. Grafton, A. Kortstee, S. Karunairetnam, T. McGhie, R. Espley, R. Hellens, and A.C. Allan. 2010. An R2R3 MYB transcription factor associated with regulation of the anthocyanin biosynthetic pathway in Rosaceae. BMC Plant Biol. 10:50.

Livak, K.J. and T.D. Schmittgen. 2001. Analysis of relative gene expression data using real-time quantitative PCR and the $2^{-\triangle \triangle C T}$ method. Methods 25:402-408.

Martin, C. 2013. The interface between plant metabolic engineering and human health. Curr. Opin. Biotechnol. 24:344-353.

Mason, G., P. Provero, A.M. Vaira, and G.P. Accotto. 2002. Estimating the number of integrations in transformed plants by quantitative real-time PCR. BMC Biotechnol. 2:1-10.

Misra, P., A. Pandey, M. Tiwari, K. Chandrashekar, O.P. Sidhu, M.H. Asif, D. Chakrabarty, P.K Singh, P.K. Trivedi, P. Nath, and R. Tuli. 2010. Modulation of transcriptome and metabolome of tobacco by Arabidopsis transcription factor, AtMYB 12, leads to insect resistance. Plant Physiol. 152:2258-2268.

Page, M., N. Sultana, K. Paszkiewicz, H. Florance, and N. Smirnoff. 2012. The influence of ascorbate on anthocyanin accumulation during high light acclimation in Arabidopsis thaliana: Further evidence for redox control of anthocyanin synthesis. Plant Cell Environ. 35:388404.

Palapol, Y., S. Ketsa, K.L. Wang, I.B. Ferguson, and A.C. Allan. 2009. A MYB transcription factor regulates anthocyanin biosynthesis in mangosteen (Garcinia mangostana L.) fruit during ripening. Planta 229:1323-1334.

Ravaglia, D., R.V. Espley, R.A. Henry-Kirk, C. Andreotti, V. Ziosi, R.P. Hellens, G. Costa, and A.C. Allan. 2013. Transcriptional regulation of flavonoid biosynthesis in nectarine (Prunus persica) by a set of R2R3 MYB transcription factors. BMC Plant Biol. 13:68.

Revilla, E. and J.M. Ryan. 2000. Analysis of several phenolic compounds with potential antioxidant properties in grape extracts and wines by highperformance liquid chromatography-photodiode array detection without sample preparation. J. Chromatography 881:461-469.
Shen, H.X., J. Zhang, Y.C. Yao, J. Tian, T.T. Song, J. Geng, and J.P. Gao. 2012. Isolation and expression of $\mathrm{McF} 3 \mathrm{H}$ gene in the leaves of crabapple. Acta Physiol. Plant. 34:1353-1361.

Tai, D.Q., J. Tian, J. Zhang, T.T. Song, and Y.C. Yao. 2014. A Malus crabapple chalcone synthase gene, $\mathrm{McCHS}$, regulates red petal color and flavonoid biosynthesis. PLoS One 10: e110570.

Takos, A.M., F.W. Jaffe, S.R. Jacob, J. Bogs, S.P. Robinson, and A.R. Walker. 2006. Lightinduced expression of a MYB gene regulates anthocyanin biosynthesis in red apples. Plant Physiol. 142:1216-1232.

Telias, A., K. Lin-Wang, D.E. Stevenson, J.M. Cooney, R.P. Hellens, A.C. Allan, E.E. Hoover, and J.M. Bradeen. 2011. Apple skin patterning is associated with differential expression of MYB10. BMC Plant Biol. 11:93-107.

Tian, J., Z. Peng, J. Zhang, T.T. Song, H.H. Wan, M.L. Zhang, and Y.C. Yao. 2015. McMYB10 regulates coloration via activating $M c F 3^{\prime} H$ and later structural genes in ever-red leaf crabapple. Plant Biotechnol. J. 13:948-961.

Tian, J., H.X. Shen, J. Zhang, T.T. Song, and Y.C. Yao. 2011. Characteristics of chalcone synthase promoters from different leaf-color malus crabapple cultivars. Sci. Hort. 129:449-458.

Velasco, R., A. Zharkikh, J. Affourtit, A. Dhingra, A. Cestaro, A. Kalyanaraman, P. Fontana, S.K. Bhatnagar, M. Troggio, D. Pruss, S. Salvi, M. Pindo, P. Baldi, S. Castelletti, M. Cavaiuolo, G. Coppola, F. Costa, V. Cova, A. Dal Ri, V. Goremykin, M. Komjanc, S. Longhi, P. Magnago, G. Malacarne, M. Malnoy, D. Micheletti, M. Moretto, M. Perazzolli, A. Si-Ammour, S. Vezzulli, E. Zini, G. Eldredge, L.M. Fitzgerald, N. Gnbutin, J. Lanchbury, T. Macalma, J.T. Mitchell, J. Reid, B. Wardell, C. Kodira, Z. Chen, B. Desany, F. Niazi, M. Palmer, T. Koepke, D. Jiwan, S. Schaeffer, V. Krishnan, C. Wu, V.T. Chu, S.T. King, J. Vick, Q. Tao, A. Mraz, A. Stormo, K. Stormo, R. Bogden, D. Ederle, A. Stella, A. Vecchietti, M.M. Kater, S. Masiero, P. Lasserre, Y. Lespinasse, A.C. Allan, V. Bus, D. Chagné, R.N. Crowhurst, A.P. Gleave, E. Lavezzo, J.A. Fawcett, S. Proost, P. Rouzé, L. Sterck, S. Toppo, B. Lazzari, R.P. Hellens, C.E. Durel, A. Gutin, R.E. Bumgarner, S.E. Gardiner, M. Skolnick, M. Egholm, Y. Van de Peer, F. Salamini, and R. Viola. 2010. The genome of the domesticated apple (Malus $\times$ domestica Borkh.). Nat. Genet. 42:833-839.

Wang, Z.G., D. Meng, A.D. Wang, T.L. Li, S.L. Jiang, P.H. Cong, and T.Z. Li. 2013. The methylation of the PcMYB10 promoter is associated with green-skinned sport in Max Red Bartlett pear. Plant Physiol. 162:885-896.

Winkel-Shirley, B. 2001. Flavonoid biosynthesis. A colorful model for genetics, biochemistry, cell biology, and biotechnology. Plant Physiol. 126:485-493.

Zhang, X.J., L.X. Wang, X.X. Chen, Y.L. Liu, R. Meng, Y.J. Wang, and Z.Y. Zhao. 2014. A and $M d M Y B 1$ allele-specific markers controlling apple (Malus x domestica Borkh.) skin color and suitability for marker-assisted selection. Genet. Mol. Res. 13:9103-9114.

Zhao, L., L.P. Gao, H.X. Wang, X.T. Chen, Y.S. Wang, H. Yang, C.L. Wei, X.C. Wan, and T. Xia. 2013. The R2R3-MYB, bHLH, WD40, and related transcription factors in flavonoid biosynthesis. Funct. Integr. Genomics 13:75-98.

Zhu, Y., K. Evans, and C. Peace. 2011. Utility testing of an apple skin color MdMYB1 marker in two progenies. Mol. Breed. 27:1-8. 